\title{
Radiation-induced segregation in W-Re: from kinetic Monte Carlo simulations to atom probe tomography experiments ${ }^{\star}$
}

\author{
Matthew J. Lloyd ${ }^{1,2, a}$, Robert G. Abernethy ${ }^{1,2}$, David E.J. Armstrong ${ }^{1}$, Paul A.J. Bagot ${ }^{1}$, \\ Michael P. Moody ${ }^{1}$, Enrique Martinez ${ }^{3}$, and Duc Nguyen-Manh ${ }^{1,2}$ \\ ${ }^{1}$ Department of Materials, University of Oxford, Parks Road, Oxford, Oxfordshire OX13PH, UK \\ ${ }^{2}$ Culham Centre for Fusion Energy, United Kingdom Atomic Energy Authority, Culham Science Centre, Abingdon, \\ Oxfordshire OX143DB, UK \\ 3 Theoretical Division, Los Alamos National Laboratory, Los Alamos, New Mexico, USA
}

Received 1 May 2019 / Received in final form 8 July 2019

Published online 21 October 2019

(C) The Author(s) 2019. This article is published with open access at Springerlink.com

\begin{abstract}
A viable fusion power station is reliant on the development of plasma facing materials that can withstand the combined effects of high temperature operation and high neutron doses. In this study we focus on $\mathrm{W}$, the most promising candidate material. Re is the primary transmutation product and has been shown to induce embrittlement through cluster formation and precipitation below its predicted solubility limit in W. We investigate the mechanism behind this using a kinetic Monte Carlo model, implemented into Stochastic Parallel PARticle Kinetic Simulator (SPPARKS) code and parameterised with a pairwise energy model for both interstitial and vacancy type defects. By introducing point defect sinks into our simulation cell, we observe the formation of Re rich clusters which have a concentration similar to that observed in ion irradiation experiments. We also compliment our computational work with atom probe tomography (APT) of ion implanted, model W-Re alloys. The segregation of Re to grain boundaries is observed in both our APT and KMC simulations.
\end{abstract}

\section{Introduction}

The realisation of fusion energy is dependant on the development of high performance plasma facing materials that can withstand the extreme operational conditions they will be subjected to. Estimates suggest that the plasma facing components (PFCs) in a prototype fusion power station (DEMO) will experience a thermal loading of $10 \mathrm{MW} \mathrm{m}^{-2}[1]$, requiring an operating temperature of around $1300 \mathrm{~K}$. W is currently the leading candidate material for these components due to its high melting temperature $(3695 \mathrm{~K})$, good thermal conductivity, resistance to sputtering and low activation under neutron irradiation [2,3]. The PFCs also receive an intense flux of $14 \mathrm{MeV}$ neutrons and high energy $\mathrm{He} / \mathrm{H}$ ions which introduce radiation damage and a significant amount of transmutation. For reactor designers, it is essential to understand the impact that these transmutation products will have on the thermal and mechanical properties of $\mathrm{W}$.

Transmutation of the W PFCs occurs through the interaction of the material with $14 \mathrm{MeV}$ neutrons produced by fusion in the reactor core, and results in an alloy of W-ReOs-Ta, the composition of which depends on the time in

\footnotetext{
* Contribution to the Topical Issue "Multiscale Materials Modeling", edited by Yoji Shibutani, Shigenobu Ogata, and Tomotsugu Shimokawa.

${ }^{a}$ e-mail: matthew.lloyd@materials.ox.ac.uk
}

the reactor. This represents a unique challenge for reactor designers, and an understanding of how precipitation occurs in these alloys is essential for predicting component lifetimes. Both the primary transmutation product, Re, and Os (which is produced in lower quantities), are produced through the neutron absorption and $\beta^{-}$decay by a $\mathrm{W}$ or Re nucleus respectively. Ta, however, is produced by neutron loss $(n, 2 n)$ reactions of $\mathrm{W}$ and subsequent $\beta^{+}$decay. At various points in the lifetime of the components, the ratios of these elements will vary [4,5], giving rise to the time dependant composition. For example, after 2 full power years exposure to an estimated DEMO neutron spectrum, FISPACT-II nuclear-inventory simulations predict that an initially pure $\mathrm{W}$ PFC will develop a concentration of 0.8 at.\% Re, 0.2 at.\% Ta and 0.05 at.\% Os. The production rate is not linear and at longer exposure times the level of Os is expected to exceed the level of $\mathrm{Ta}$ [4]. In addition, spatial inhomogeneities in the neutron flux and localised moderation of the neutrons leads to enhancement of transmutation at specific locations around the reactor, such as near water coolant pipes [5].

At present, there is no facility capable of reproducing the extremes of neutron dose and temperature that PFCs will receive in DEMO. Because of this, there is a lack of experimental evidence on neutron irradiation under reactor relevant conditions. Heavy ion $[6,7]$, proton [8], and fission spectrum neutron irradiation campaigns [9-13] have been used to simulate neutron damage in W 
and W-Re-Os. Ion implantation does not induce transmutation but can be used to introduce radiation damage into model alloys. This has advantages in that it allows a wide range of compositions to be studied easily with controlled irradiation conditions. However, transmutation occurs simultaneously to the introduction of radiation damage, and may produce different behaviour.

$\mathrm{Xu}$ et al. investigated binary $\mathrm{W}-2 \mathrm{Re}$ alloys and ternary $\mathrm{W}-2$ Re-1Os/W-2Re-1Ta alloys, ion irradiated using W self ions to $33 \mathrm{dpa}$, using APT [6,7]. Clear precipitation of Re was seen at 573 and $773 \mathrm{~K}$, with Os acting to suppress the precipitation of Re by preferentially forming small Os rich clusters, $1.9 \mathrm{~nm}$ average diameter compared to $3.3 \mathrm{~nm}$ in the binary alloy. Though similar in composition to the predicted transmutation levels, Xu et al.'s work focused on temperatures lower than the planned operating temperature of $\mathrm{W}$ in a reactor. In addition to this, a consensus has not yet been reached on the use of ions to simulate neutron damage. Recent studies of neutron irradiated W have demonstrated the importance of voids on the formation of Re precipitates, something that is not reproduced in ion irradiation [13].

Irradiation using fission spectrum neutrons is the closest alternative to direct fusion neutron exposure, in terms of the damage mechanism and introduction of transmutation products. Several neutron irradiation campaigns have been conducted since the 1990s in a range of different reactors. Hasegawa et al. have carried out irradiations at a range of temperatures and doses at JOYO, HFIR and JMTR, summarised in a schematic diagram of the damage produced at these conditions [10]. Void and precipitate formation has been shown to be favoured at higher temperatures and doses. Recent work by $\mathrm{Hu}$ et al. has used High Angle Annular Dark Field (HAADF) imaging and Scanning Transmission Electron Microscopy (STEM) EDX mapping to study transmutation induced precipitation in more detail [12]. Clear precipitation was observed with Re and Os forming lenticular precipitates and decorating grain boundaries. Os was seen to form in the centre of these clusters and voids were shown to be associated with precipitates. Despite the benefits of using neutron irradiation to study these materials, the higher thermal neutron flux from a fission reactor introduces a much higher concentration of transmutation products, particularly Os, than is predicted for the PFCs in DEMO. An Os content as low as 0.1 at.\% has been shown to impact the structure of Re clusters [13], highlighting the importance of using realistic quantities of Re/Os in these studies. In addition to this, temperature control and quantification can be an issue during neutron irradiation experiments. The irradiation temperatures used in the irradiation experiments discussed above were also lower than the operating temperature of $\mathrm{W}$ in a reactor (maximum of $800^{\circ} \mathrm{C}$ ).

Klimenkov et al. studied the precipitation behaviour in more representative alloy compositions using W samples irradiated as part of the Extremat II program [9]. Because of its proximity to a sample with a high thermal neutron absorption cross section, the sample received a neutron dose of predominantly fast neutrons. Because of this, transmutation of the sample resulted in a
W-1.4Re-0.1Os-0.03Ta alloy [13], which is closer to the level predicted by FISPACT-II modelling [4]. STEM-EDX was used to clearly show Re decoration of grain boundaries and the formation small precipitates in the centres of grains. No Os segregation to the grain boundaries was seen in the polycrystalline material but in the single crystal, a small amount of signal from Os was correlated with the observed Re clusters. Further characterisation of these samples using a combination of APT and STEM-EDX has shown that voids formed during irradiation are decorated with both Re and Os. The Os appears to form a small cluster at the void which is surrounded with a shell of Re [13]. The process through which these precipitates form is still not clear, with different mechanisms proposed to explain their formation.

Experimental studies such as those discussed previously can only capture a snapshot of the material following irradiation, but cannot always explain the process through which precipitation has occurred. Computational models can provide access to time and length scales inaccessible to experiments and give insight into these processes. In $\mathrm{Fe}-\mathrm{Cr}$, on-lattice atomistic kinetic monte carlo (KMC) models have been used extensively to study the radiation induced precipitation of $\mathrm{Cr}$. These rigid lattice models use Density Functional Theory (DFT) simulations to provide interaction parameters, based on a pairwise broken-bond energy model [14-17]. By including the solute concentration dependency to these pairwise parameters, defect migration in alloy systems can be studied, providing good agreement with nanoscale experimental characterisation techniques such as APT [16]. More recently, these models have been extended to include both vacancy and interstitial defect types, and used to study ideal defect sinks [17] and the role of defect sink density on the precipitation of $\mathrm{Cr}$ in $\mathrm{Fe}-\mathrm{Cr}$ alloys [18].

The W-Re binary system is less extensively studied than $\mathrm{Fe}-\mathrm{Cr}$, however, recent studies have used a similar on-lattice approach, with a DFT parameterised pairwise energy model, and solute concentration dependant interactions [19]. In W-Re, the mixed interstitial dumbbell is thought to be of particular importance, as unlike the selfinterstitial which is confined to $1 \mathrm{D}$ motion, the mixed interstitial is able to move through a series of rotations and translations, to effectively migrate in 3D [20-22]. This increased mobility of the mixed interstitial has been shown to facilitate the nucleation and growth of Re clusters in KMC models, despite the predicted solid solution. The pairwise binding energy of the di-vacancy in this parameterisation is close to zero, meaning that void nucleation is not observed in KMC simulations. However, cluster nucleation and growth is facilitated by the clustering of mixed interstitial defects with Re solute, to form clusters up to $50 \%$ Re at their centre [19]. Though this may explain the clustering seen in ion irradiation studies, more recent APT experiments have shown void decoration is also a key effect in these alloys [13].

To overcome the potential underestimation of the vacancy binding in the pairwise $\mathrm{W}-\mathrm{Re}$ model, cluster expansion (CE) techniques have been used to create a Hamiltonian that allows void formation, by considering the interactions between 3 or more atoms/defects 
Table 1. Possible events within the KMC and SGMC models. Lattice sites are designated as follows: V=Vacancy, $\mathrm{A}, \mathrm{B}=$ Atoms, $\mathrm{AA}=\mathrm{SIA}, \mathrm{AB}=$ Mixed Interstitial, $\mathrm{S}=$ Sink Site.

\begin{tabular}{lllll}
\hline & Vacancies & Interstitals & Instantaneous & Deposition \\
\hline KMC & $\mathrm{V}+\mathrm{A} \rightarrow \mathrm{A}+\mathrm{V}$ & $\mathrm{AA}+\mathrm{A} \rightarrow \mathrm{A}+\mathrm{AA}$ & $\mathrm{AA}+\mathrm{V} \rightarrow \mathrm{A}+\mathrm{A}$ & $\mathrm{A} \rightarrow \mathrm{AA}+\mathrm{V}$ \\
& $\mathrm{V}+\mathrm{B} \rightarrow \mathrm{B}+\mathrm{V}$ & $\mathrm{AB}+\mathrm{A} \rightarrow \mathrm{A}+\mathrm{AB}$ & $\mathrm{AB}+\mathrm{V} \rightarrow \mathrm{A}+\mathrm{B}$ & $\mathrm{B} \rightarrow \mathrm{AB}+\mathrm{V}$ \\
& & $\mathrm{AB}+\mathrm{B} \rightarrow \mathrm{B}+\mathrm{AB}$ & $\mathrm{AA}+\mathrm{S} \rightarrow \mathrm{A}+\mathrm{S}$ & \\
& & $\mathrm{AA}\langle 111\rangle \rightarrow \mathrm{AA}\langle 111\rangle$ & $\mathrm{V}+\mathrm{S} \rightarrow \mathrm{A}+\mathrm{S}$ & \\
& $\mathrm{AB}\langle 111\rangle \rightarrow \mathrm{AB}\langle 111\rangle$ & & \\
& & & $\mathrm{A}+\mathrm{S} \rightarrow \mathrm{B}+\mathrm{S}$ \\
$\mathrm{SGMC}$ & $\mathrm{V}+\mathrm{A} \rightarrow \mathrm{A}+\mathrm{V}$ & $\mathrm{AA}+\mathrm{A} \rightarrow \mathrm{A}+\mathrm{AA}$ & & $\mathrm{B} \rightarrow \mathrm{A}$ \\
& $\mathrm{V}+\mathrm{B} \rightarrow \mathrm{B}+\mathrm{V}$ & $\mathrm{AB}+\mathrm{A} \rightarrow \mathrm{A}+\mathrm{AB}$ & & \\
& & $\mathrm{AB}+\mathrm{B} \rightarrow \mathrm{B}+\mathrm{AB}$ & & \\
\hline
\end{tabular}

at once. At high temperatures (stage IV Recovery [3]) vacancies become much more mobile and play a more significant role in microstructural evolution. Monte Carlo (MC) simulations using a CE Hamiltonian have predicted the precipitation of Re in $\mathrm{W}$ alloys through the formation of small voids, decorated with Re atoms [23].

In this research, we present an atomistic KMC model containing both vacancy and interstitial defect types (self and mixed interstitial dumbbells), as well as solute concentration dependant interactions. The model is built on the open source Stochastic Parallel PARticle Kinetic Simulator (SPPARKS) [24], and uses a pairwise energy model parameterised for the W-Re binary system using DFT simulations [19]. In KMC simulations of W-2Re and W-1.4Re we observe the decoration of point sinks and planar sinks with Re solute atoms. We go on to compare KMC simulations with experimental evidence of grain boundary segregation and cluster formation in self-ion implanted W-1.4\%Re. These experimental observations are made using targeted Atom Probe Tomography (APT).

\section{Computational methodology}

The basis of the model presented here is the Monte Carlo technique, which relies on the generation of random numbers to sample a distribution of possible events. For the study of the diffusion of defects, the difference in the energy, $\Delta E_{i \rightarrow j}$, between two states, $i$ and $j$ is used to generate the probability of a particular rate occurring. To construct this change in energy between states, we rely on pairwise energies, obtained using DFT. These pairwise potentials, $\varepsilon$, are summed for the initial and final state to produce a total initial and final energy, according to a broken-bond model [14-17].

In this research we utilise this change in energy in two ways. The first is within a Semi Grand Canonical MC (SGMC) model, in which the equilibrium composition of Re is obtained for a given chemical potential in the solid solution phase. The second uses the energy difference between the saddle point and the minimum of each transition to calculate the rate of a particular event, relying on harmonic transition state theory, facilitating Kinetic MC (KMC) simulations.

The model we present in this study is implemented into the open source Stochastic Parallel PARticle Kinetic
Simulator (SPPARKS) code. We include 3 types of points defects: vacancies, self-interstitial dumbbells and mixed interstitial dumbbells. We do not currently consider the Re-Re interstitial dumbbell as they are less commonly occurring [19]. Simulated results are visualised using The Open Visualization Tool (OVITO) [25]. The processes implemented in the model are summarised in Table 1.

\subsection{Semi-Grand Canonical Monte Carlo}

In the SGMC model, atoms are exchanged from a reservoir, using a probability which is given by the change in energy before and after the swap, $\Delta E_{i \rightarrow j}$. Atoms are selected at random from the box. If a $\mathrm{W}$ atom is selected it is swapped to a Re atom and vice versa, with a probability $\prod_{i \rightarrow j}$, given by equation (1), where $\Delta N_{\mathrm{Re}}$ is the change in the number of Re atoms, $\Delta \mu$ is the change in chemical potential, and $T$ is the temperature

$$
\prod_{i \rightarrow j}=\min \left\{1, \quad \exp \left(\frac{-\Delta E_{i \rightarrow j}-\Delta \mu \Delta N_{\mathrm{Re}}}{k_{\mathrm{B}} T}\right)\right\} .
$$

For a fixed temperature and chemical potential, $\Delta \mu$, the concentration of Re within the box is allowed to fluctuate through swaps with the reservoir until the system reaches equilibrium. Inside a miscibility gap where a mixture of phases are present, the change in chemical potential does not determine the composition of the system. The system is therefore not stable within these regions and jumps to a higher concentration. By plotting the change in chemical potential, $\Delta \mu$ against the equilibrium concentration of Re, the miscibility gap is shown as a step in the plot.

By starting simulations with a concentration of defects, the equilibrium configuration of the system with a given chemical potential and temperature can be obtained. Defects are allowed to exchange with atoms, based on a probability given by equation (2), where $\Delta E_{i j}$ in this case is the change in energy before and after the jump

$$
\prod_{i \rightarrow j}=\min \left\{1, \quad \exp \left(-\frac{\Delta E_{i \rightarrow j}}{k_{\mathrm{B}} T}\right)\right\} .
$$


Evolving the system with a fixed concentration of defects allows us to study the equilibrium configuration of the defects within the box for a given Re concentration.

\subsection{Kinetic Monte Carlo}

\subsubsection{Defect migration}

The SGMC algorithm can provide insight into the equilibrium configuration of a particular defect and solute concentration, but in order to study the Re clustering/ precipitation kinetics, a KMC model must be used.

The KMC model relies on the calcualtion of process rates, which are calulated using harmonic transition state theory (TST). The change in energy between states $i$ and $j$ (the initial state and saddle point between initial and final states), $\Delta E_{i j}$, is used to generate the rate, $\lambda_{i \rightarrow j}$, by multiplying the attempt frequency, $\nu$, as shown in equation (3)

$$
\lambda_{i \rightarrow j}=\nu \cdot \exp \left(-\frac{\Delta E_{i \rightarrow j}}{k_{\mathrm{B}} T}\right) .
$$

The attempt frequency, $\nu$ depends on the type of defect: $\nu_{V}=6.5 \times 10^{12} \mathrm{~Hz}$ for vacancies and $\nu_{I}=1.5 \times 10^{12} \mathrm{~Hz}$ for interstitials [19]. We use this expression to approximate the rates associated with all defect types within our model (vacancies, self-interstitials and mixed interstitials). Interstitials migrate quickly as the activation energy for their movement and rotation is very low compared to the vacancies, making it closer to $k T$. TST is valid for events with high activation energies such as the vacancies, but when this barrier is lower (such as for interstitials) the validity of this approach is more questionable due to the increased probability of tunnelling through the barrier.

The calculation of the change in energy, $\Delta E_{i j}$, varies for different defects. There are different migration pathways for vacancies, self-interstitials and mixed interstitials. For vacancies we consider the energetics of the defect at the saddle point. As shown in equation (4), the energy is constructed using the sum of the pairwise energies, $\varepsilon$, according to the so-called broken-bond model

$$
\Delta E_{i \rightarrow j}^{V}=\sum_{\theta} \varepsilon_{\alpha-\theta}^{(\mathrm{s})}-\left(\sum_{\beta} \varepsilon_{\alpha-\beta}^{(n)}+\sum_{\gamma \neq \alpha} \varepsilon_{\delta-\gamma}^{(n)}\right)+\Delta E_{i \rightarrow j}^{x} .
$$

The first term on the RHS of equation (4) is the sum of the pairwise interactions at the saddle point, $\alpha$, over the neighbours of the atom at the saddle point, $\theta$, where $s$ is the neighbour shell considered which in our model extends to $2 \mathrm{NN}$. The second term is the energy of the original state, $i$, and is made up of two sums: the energy of the atom at its original position with its neighbours, and the sum of the defects at its original position with its neighbours. The sum is made over that bonds between the atom, $\alpha$ and its neighbours, $\beta$. For the vacancy, $\delta$ this sum is made over its neighbours, $\gamma$ not including the bond that was already counted in the first sum, where $\gamma=\alpha$. The final term is the contribution to the change in energy due to the change in the concentration of solute, $\Delta E_{i \rightarrow j}^{x}$, which depends on the change in the solute concentration, $x$ between states $i$ and $j$. This is because some of the pairwise interactions, $\varepsilon$ depends on the local concentration and must be recalculated.

For the interstitial, the change in energy is is approximated between the initial and final positions. The overall change in energy between states $i$ and $j$ for an interstitial jump, $\Delta E_{i \rightarrow j}$ is given by equation (5), where $E_{m}$ is a constant migration energy and $\Delta \mathscr{H}_{i \rightarrow j}$ is the change in energy between states

$$
\Delta E_{i \rightarrow j}= \begin{cases}E_{m}+\Delta \mathscr{H}_{i \rightarrow j} & \text { if } \Delta \mathscr{H}_{i \rightarrow j}>0 \\ E_{m} & \text { if } \Delta \mathscr{H}_{i \rightarrow j}<0 .\end{cases}
$$

If $\Delta \mathscr{H}_{i \rightarrow j}<0$, the overall change in energy used to calculate the rate, $\Delta E_{i \rightarrow j}$ is simply set to $E_{m}^{\mathrm{SIA}}$ or $E_{m}^{\mathrm{MI}}$ for the self-interstitial or mixed interstitial respectively. For the interstitials, the change in the energy between initial and final states, $\Delta \mathscr{H}_{i \rightarrow j}$, is simply approximated as the difference between the initial and final states, rather than considering the defect at the saddle point. For the case of the self interstitial, the change in energy is given by equation (6) where $E_{m}^{\mathrm{SIA}}=0.003 \mathrm{eV}$ [19] for the atom $\alpha$ and its neighbours $\beta$ and for the interstitial $\delta$ and its neighbours $\gamma$ excluding the case where $\delta=\gamma$.

$$
\begin{aligned}
\Delta E_{i \rightarrow j}= & E_{m}^{\mathrm{SIA}}-\left(\sum_{\beta} \varepsilon_{\alpha-\beta}^{(n)}+\sum_{\gamma \neq \alpha} \varepsilon_{\delta-\gamma}^{(n)}\right) \\
& -\left(\sum_{\beta} \varepsilon_{\delta-\beta}^{(n)}+\sum_{\gamma \neq \delta} \varepsilon_{\alpha-\gamma}^{(n)}\right)+\Delta E_{i \rightarrow j}^{x} .
\end{aligned}
$$

The self interstitial migrates along a set $\langle 111\rangle$ direction, chosen at random when it is inserted into the simulation cell. In addition to its migration, the SIA has a probability of rotating to a new $\langle 111\rangle$ direction with a probability given by equation (3) where the change in energy is a fixed activation energy for rotation, $E_{R}^{\mathrm{SIA}}=0.43$ [19].

For the mixed-interstitial, the migration occurs through a series of translations and rotations, as described by Suzudo et al. [20]. To implement this migration process into the KMC model, we assume the mixed interstitial migrates via an initial translation in the $\langle 111\rangle$ direction, followed by a rotation and a second $\langle 111\rangle$ translation. This results in an effective jump in the $\langle 100\rangle$ direction, relative to its original position, as shown schematically in Figures 1 and 2 .

To calculate the total migration rate, $\lambda_{T}$, of this process we employ equation (7), where the time associated with a process, $t^{i}$, is given by $1 / \lambda^{i}$. The total time of the process, $t_{\langle 100\rangle}^{a \rightarrow d}$, is given by the sums of the times associated with each translation and the rotation, as shown in equation (7)

$$
t_{\langle 100\rangle}^{a \rightarrow d}=t_{\langle 111\rangle}^{a \rightarrow b}+t_{r}^{b \rightarrow c}+t_{\langle 111\rangle}^{c \rightarrow d} .
$$

The total rate, $\lambda_{T}$, is therefore simply the inverse of the total time and is given by equation (8). The rate of each translation can be calculated using equation (3) where the 


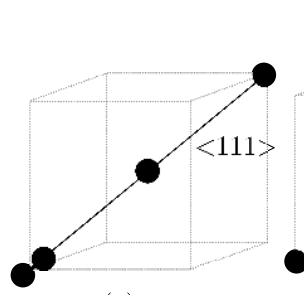

(a)

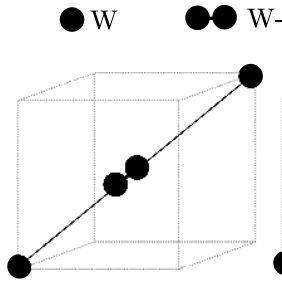

(b)

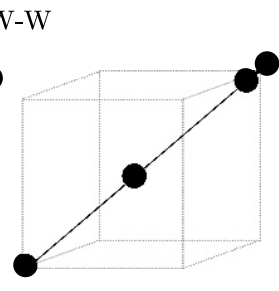

(c)
Fig. 1. Schematic diagram of the migration pathway for a self interstitial dumbbell in $\mathrm{W}$, oriented along the [111] direction. Reproduced from [20].

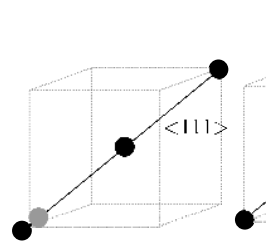

(a) (b)

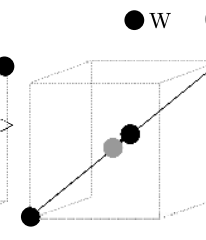

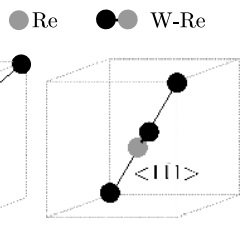

(c)

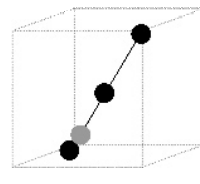

(d)
Fig. 2. Schematic diagram showing the migration pathway for the W-Re mixed interstitial dumbbell in W. In the case of the mixed dumbbell, migration occurs through a series of rotation and translation events, which from (a) to (d) effectively results in a migration along the [100] direction. Reproduced from [20].

change in energy for each translation in the process is calculated using equation (6) using the migration barrier for the mixed interstitial $E_{m}^{12}=0.12 \mathrm{eV}$ [19]. For the rotation of the mixed interstitial dumbbell, a fixed activation energy, $E_{R}^{\mathrm{MI}}=0.03 \mathrm{eV}$, is used

$$
\lambda_{T}=\frac{1}{\left(1 / \lambda_{\langle 100\rangle}^{a \rightarrow c}\right)+\left(1 / \lambda_{r}\right)+\left(1 / \lambda_{\langle 111\rangle}^{b \rightarrow c}\right)} .
$$

\subsubsection{Instantaneous process and deposition}

Other events that occur within the KMC simulations are the recombination of defects, either with each other or at a sink, and the deposition of defects into the simulation box. When a vacancy and an interstitial come within third nearest neighbours ( $3 \mathrm{NN}$ ) of each other, they recombine instantaneously leaving behind either two $\mathrm{W}$ atoms or a $\mathrm{W}$ and a Re atom depending on the interstitial type ( $\mathrm{W}-\mathrm{W}$ or W-Re respectively). When a defect comes within $3 \mathrm{NN}$ of a sink, it is also instantaneously recombined. For a vacancy this leaves behind a W atom, and for interstitials this results in either a $\mathrm{W}$ atom or a Re atom.

Because of the attractive binding energy between the SIA and a Re atom, we assume the transition of a SIA to a MI dumbbell to be instantaneous whenever a SIA comes within $3 \mathrm{NN}$ of a Re atom. The deposition of defects inserts a Frenkel pair into the simulation box. These defects are inserted with a separation distance $2 a$ where $a$ is the lattice parameter. If the defects are within $3 \mathrm{NN}$ range, they are instantaneously recombined. This process occurs for a set deposition rate of $N$ pairs per second. The inserted interstitial type depends on the site that is selected. A site is chosen at random within the box. If this site is a Re atom, a MI is inserted, and if it is a W a SIA is inserted, ensuring that solute concentration is conserved throughout the simulation.

\section{Computational results}

\subsection{SGMC}

Figure 3 shows the equilibriated Re content as a function of the change in chemical potential, $\Delta \mu$. There is a jump in the concentration at $100 \mathrm{~K}$ indicating the presence of a miscibility gap, implying a phase transformation is predicted by this parameterisation. This also implies that the higher temperature simulations may need to be extended for much greater times in order to achieve equilibrium as no transformation is seen at these temperatures in Figure 3.

As can be seen in Figure 4, the introduction of point defects into the simulation, the clustering of Re can occur through the interaction of the solute with the defects. The simulations shown in Figure 4 were carried out in a $2 \times 30 \times 30 \times 30$ atom box, at $600 \mathrm{~K}$ using a fixed defect concentration of $0.5 \%$ Vacancies and $0.1 \%$ Mixed interstitials, and a Re concentration of $4 \% \mathrm{Re}$ and $0.5 \% \mathrm{Re}$ respectively. In the case of Figure $4 \mathrm{~b}$, the clustering of mixed interstitials is observed along with the attachment of Re to these mixed interstitial clusters. This is to be expected as the mixed interstitials have an attractive binding energy to one another $(-3.2 \mathrm{eV}[19])$, and a lower but still attractive binding to the Re atoms $(-0.53 \mathrm{eV}[19])$. In the case of Figure $4 \mathrm{a}$ however, there is no direct binding of the vacancies to one another, as is seen in Figure $4 \mathrm{~b}$. In this parameterisation, the vacancies only have a weak attraction to one another and thus do not cluster together. In Figure 4a, the clustering of vacancies is facilitated by the formation of an alternating structure of Re and vacancies. This is due to the fact that the binding energy between the vacancies and Re is more attractive $(-0.2096 \mathrm{eV}[19])$ than the binding between two vacancies $(-0.0146 \mathrm{eV}[19])$.

\subsection{Kinetic Monte Carlo}

\subsubsection{Calculation of diffusion coefficients}

The diffusion coefficient for defects can be expressed as an Arrhenius equation as shown in equation (9), where $E_{A}$ is the activation energy for migration, $D_{0}$ is the diffusion prefactor and $T$ is the temperature

$$
D=D_{0} \exp \left(-\frac{E_{A}}{k T}\right)
$$

The diffusion of defects is affected by the presence of Re which slows down the migration. Local variations in the solute concentration can limit the migration of defects by trapping them. To quantify the effect that this has on the migration of defects within the model, the diffusion coefficient can be plotted as a function of Re concentration within the simulation box. The diffusion coefficient, $D$ can 


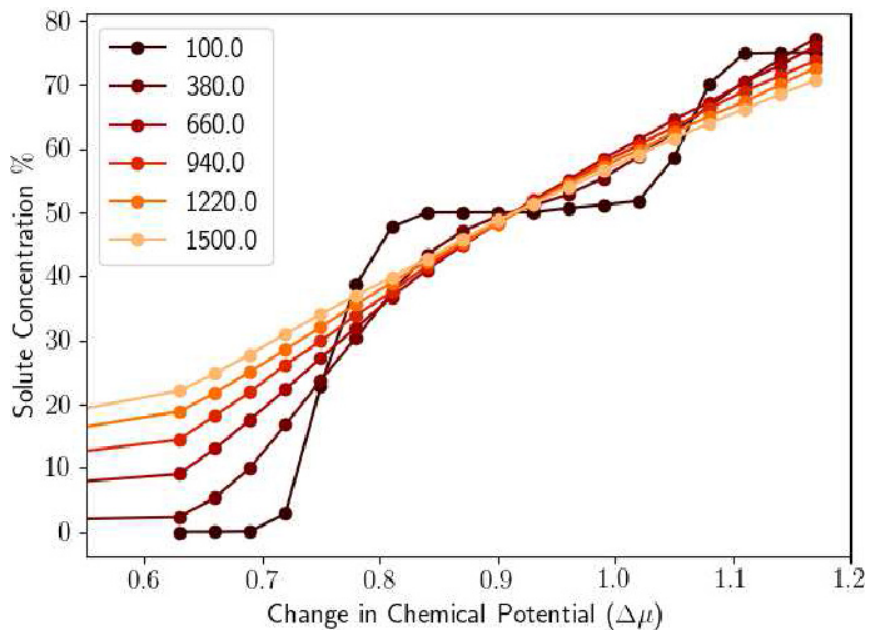

Fig. 3. Plot of equilibrated solute concentration in $\%$ as a function of the change in chemical potential $\Delta \mu$ for a range of temperatures.

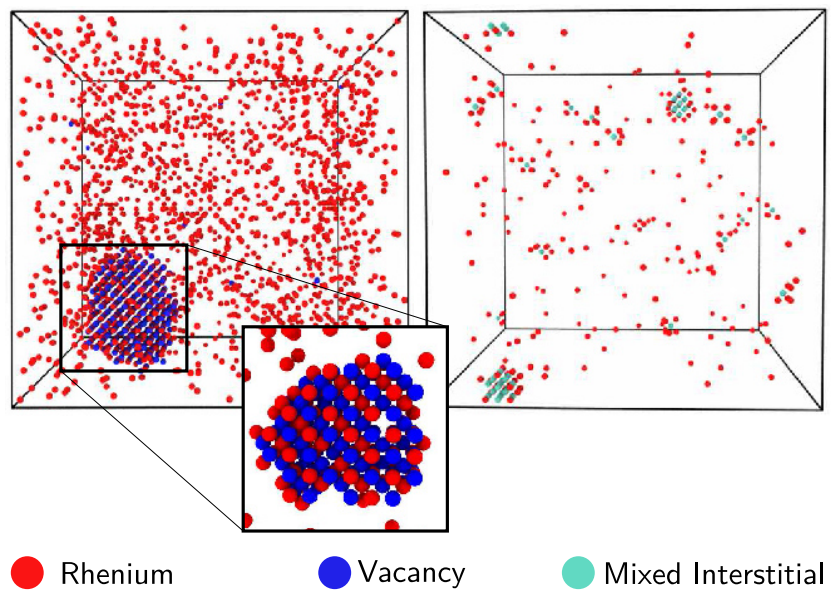

Fig. 4. Equilibriated configurations of W-Re-Vacancy and W-Re-Interstitial for a fixed starting defect concentration and change in chemical potential, $\Delta \mu$.

also be given by equation (10):

$$
\left\langle\Delta r^{2}\right\rangle=6 D \Delta t
$$

where $\left\langle\Delta r^{2}\right\rangle$ is the mean-squared-displacement and $\Delta t$ is the time. To calculate the value of the diffusion coefficient, the trajectory of a defect or solute atom is output as a function of time. The trajectory is subdivided into different time steps, $\Delta t^{(i)}$ and the displacement that occurred during this timestep, $\Delta r^{(i)}$ is measured. The gradient of a linear fit of the square displacement vs. time steps, $\Delta t^{(i)}$ divided by 6 gives the value of the diffusion coefficient.

The timestep in the KMC simulation, $\Delta t_{\mathrm{kmc}}$ is rescaled by the equilibrium vacancy concentration, $C_{\mathrm{eq}}$, for simulations of solute diffusion. Typical vacancy concentrations are many orders of magnitude lower than can be simulated in a cell and so the timestep has to be rescaled for simulations of solute or self diffusion. The rescaled timestep,

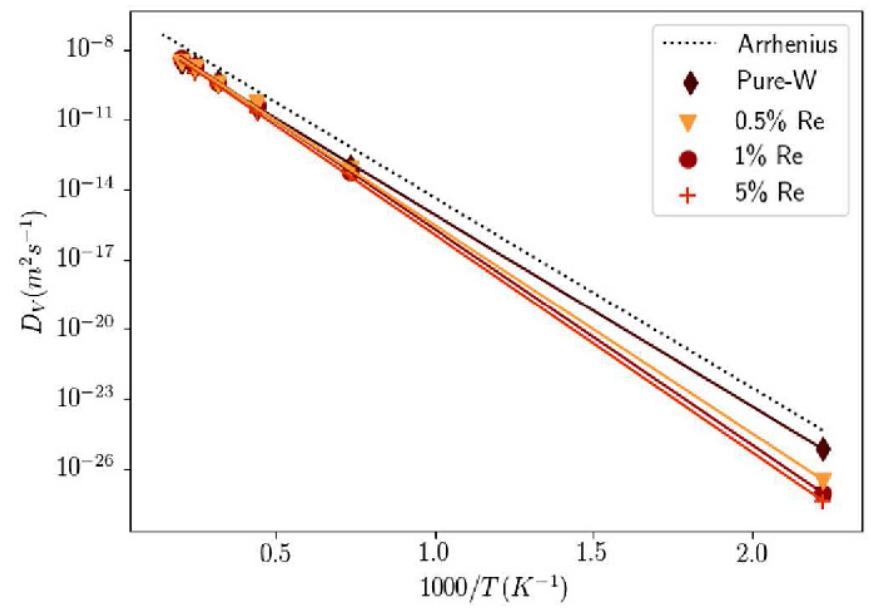

Fig. 5. Vacancy diffusivity, $D_{v}$, as a function of temperature and Re concentration. Dashed line shows the Arrhenius relationship described by equation (9).

$\Delta t$, is given by equation (11)

$$
\Delta t=\Delta t \frac{C}{C_{\mathrm{eq}}} .
$$

The vacancy concentration in the simulation cell, $C$ is simply given by $1 / N$ for simulations containing a single vacancy. The equilibrium vacancy concentration, $C_{\text {eq }}$, calculated using equation (12) where the formation energy of the vacancy, $E_{F}^{V}$, is recalculated depending on the local environment

$$
C_{\text {eq }}=\exp \left(-\frac{E_{F}^{V}}{k T}\right)
$$

The instantaneous vacancy formation energy, $E_{F}^{V}$, is given by equation (13) and depends on the local environment of the defect. The sum of the first two terms on the RHS of equation (13) gives the cohesive energy of pure $\mathrm{W}$. The sum of the third and fourth terms on the RHS of equation (13) are the local binding of the vacancy to its neighbours, and is the sum over the $z_{1}$ first nearest and $z_{2}$ second nearest neighbours multiplied by the pairwise energies, $\varepsilon$, which depend on the local solute environment. The subtraction of these two gives the vacancy formation energy as a function of the local alloy and can be used to estimate the equilibrium vacancy concentration, $C_{\mathrm{eq}}$

$$
E_{F}^{V}=-\frac{z_{1}}{2} \varepsilon^{(i)}-\frac{z_{2}}{2} \varepsilon^{(i)}-z_{1} \varepsilon^{(i)}-z_{2} \varepsilon^{(i)} .
$$

Figure 5 shows the vacancy diffusivity as a function of temperature and the solute concentration. Table 2 shows the diffusion coefficient, $D_{0}$ and the activation energy, $E_{A}$ for each of the fitted lines. Figure 5 shows that the motion of the vacancies is slowed by the presence of Re in the simulation cell. Vacancies have an attractive binding to Re [19] and therefore are slowed by Re that they encounter in the box. 
Table 2. Diffusion coefficient, $D_{0}$ and activation energy, $E_{A}$ for vacancy diffusion as a function of solute concentration, $x$.

\begin{tabular}{lll}
\hline$x_{\text {Re }}(\%)$ & $D_{0}\left(\mathrm{~m}^{2} \mathrm{~s}^{-1}\right)$ & $E_{A}(\mathrm{eV})$ \\
\hline 0.0 & $1.4 \times 10^{-7}$ & 1.64 \\
0.5 & $2.61 \times 10^{-7}$ & 1.78 \\
1.0 & $3.59 \times 10^{-7}$ & 1.84 \\
5.0 & $2.56 \times 10^{-7}$ & 1.86 \\
\hline
\end{tabular}

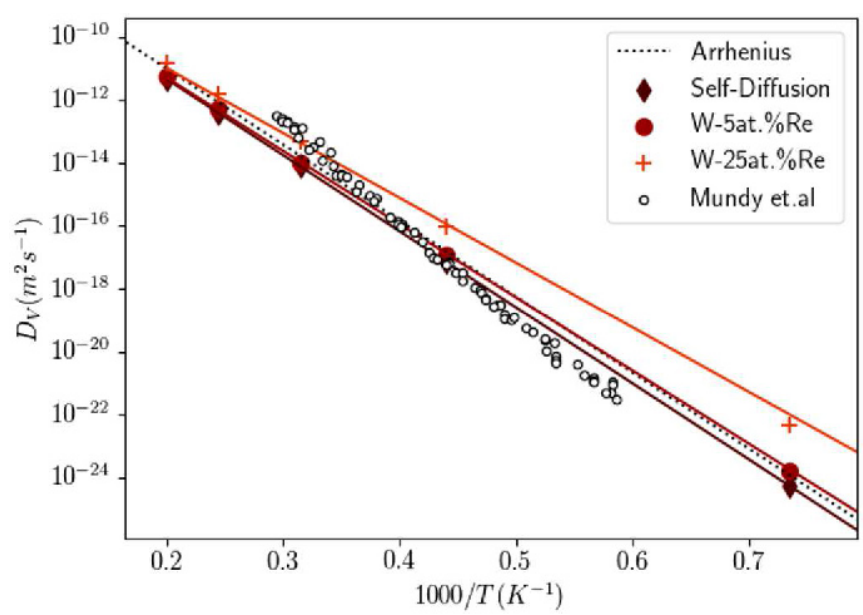

Fig. 6. Solute and self-diffusivity, $D_{s}$, as a function of temperature and Re concentration. Dashed line shows the Arrhenius relationship described by equation (9) using an activation energy of $4.79 \mathrm{eV}$. The black points represent experimental values for the self-diffusion of $\mathrm{W}$ in $\mathrm{W}$ taken from [26].

Table 3. Diffusion coefficient, $D_{0}$ and activation energy, $E_{A}$ for solute diffusion as a function of solute concentration, $x$.

\begin{tabular}{lll}
\hline$x_{\operatorname{Re}}(\%)$ & $D_{0}\left(\mathrm{~m}^{2} \mathrm{~s}^{-1}\right)$ & $E_{A}(\mathrm{eV})$ \\
\hline Self-diffusion equation $(9)$ & $6.49 \times 10^{-7}$ & 4.790 \\
0.0 & $3.03 \times 10^{-7}$ & 4.79 \\
5.0 & $2.20 \times 10^{-7}$ & 4.61 \\
25.0 & $1.27 \times 10^{-7}$ & 4.08 \\
\hline
\end{tabular}

Figure 6 shows the average solute diffusivity, $D_{s}$ as a function of temperature and solute concentration as well as the self-diffusion of $\mathrm{W}$ in $\mathrm{W}$. The dashed line on Figure 6 represents the Arrhenius relationship described by equation (9) using an activation energy of $4.79 \mathrm{eV}$ [19]. The hollow circles represent experimental data points taken by Mundy et al. [26] for the self-diffusion of W. The diamond shaped markers represent the self diffusion calculated from our simulations. The other markers show the diffusivity of the solute atoms. The values for the solute diffusivity are in good agreement with both the experimental data points and that predicted by equation (9). The introduction of $5 \%$ Re slightly decreases the solute migration activation energy as shown in Table 3 which becomes more pronounced at $25 \%$ Re. This also agrees well with Huang et al's work using this parameterisation [19].
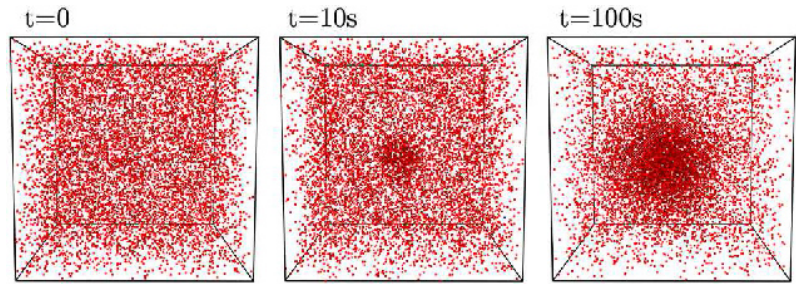

Fig. 7. Precipitation of Re around a point sink at 10 and $100 \mathrm{~s}$ of irradiation in a W-2at.\%Re alloy. A dose rate of $1 \times 10^{-3} \mathrm{dpa} / \mathrm{s}$ was used at an irradiation temperature of $1800 \mathrm{~K}$ which ensures that vacancies are mobile within our simulation.

\subsubsection{Radiation-induced segregation and precipitation}

To study the formation of Re precipitates under irradiation, we focus on a W-2Re alloy as precipitation in dilute alloys such as this has been observed both experimentally $[6,7]$ and computationally [19]. The simulation is started with a central ideal point sink. If any defects come within range of this sink, they are removed according to the processes described in Table 1. Figure 7 shows the position of Re atoms at $0 \mathrm{~s}, 10 \mathrm{~s}$ and $100 \mathrm{~s}$. The model predicts the formation of a Re rich cluster around the point sink in the centre of the simulation cell. This implies that there is a flux of defects towards this sink which transport Re towards it, resulting in the formation of the Re cluster.

In the case where no sinks are included in the simulation cell, no Re cluster formation is observed. If the simulation is started with a Re cluster and no sink, the dissolution of Re is observed. The mechanism proposed by Huang et al. suggests that the immobilisation of the mixed interstitials by Re or by other mixed interstitials provides the vacancy sufficient time to migrate to the trapped dumbbell and recombine. This means that the trapped interstitial effectively acts as the sink that we have introduced artificially in the simulation shown in Figure 7. However, using our model we observe that the interstitial, upon reaching a Re rich area, first becomes trapped in that region as suggested, but then continues to dissolve the cluster over time. In the time it takes the vacancy to reach the immobilised interstitial, it has sufficient time to dissolve the cluster slightly, reducing the central Re concentration and reducing the effectiveness of the cluster to trap further interstitials, by spreading out the interface between $\mathrm{W}$ and Re. Over many of these events the cluster reaches a point at which it is too diffuse to act as a trapping site and it is dissolved back into the matrix.

Figure 8 shows the Re concentration in the cluster shown in Figure 7 as a function of radius from the point sink. The concentration is estimated using a series of progressively smaller concentric spherical shells. Plotted as hollow squares is the radial concentration profile seen by $\mathrm{Xu}$ et al. using APT of a W-2Re alloy, irradiated to $33 \mathrm{dpa}$ at $773 \mathrm{~K}$ using self ion implantation. As seen in Figure 8 , when a sink is artificially introduced into the box, there is strong Re segregation. After $100 \mathrm{~s}$ of irradiation the maximum concentration of $\mathrm{Re}$ at the sink is between 25 and 30 at.\%. This agrees well with experimental data from Xu et al.'s ion implantation which shows a 


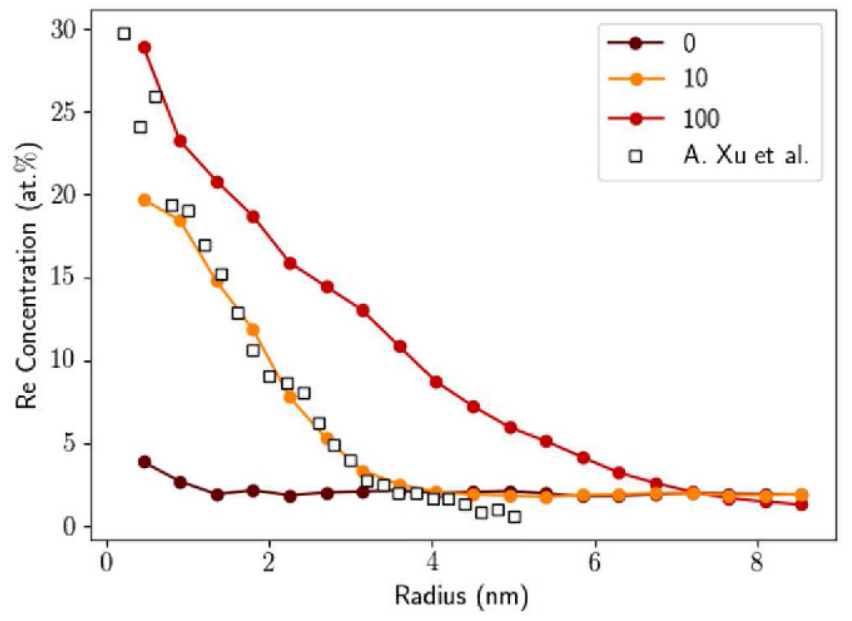

Fig. 8. Re concentration as a function of radius from an ideal point sink, in a $\mathrm{W}-2 \%$ Re alloy irradiated with a dose rate of $1 \times 10^{-3} \mathrm{dpa} / \mathrm{s}$ and an irradiation temperature of $1800 \mathrm{~K}$. Legend shows simulated time in seconds.

maximum cluster concentration of around 30 at.\% [6]. We do not see the saturation of the Re content at $50 \%$ in the centre of the precipitate but overall it is lower than the level predicted by Huang et al. [19]. This could be due in part to the increased cluster dissolution that we see in our model. The Re profile at $10 \mathrm{~s}$ shown in Figure 8 is very similar to he experimental profile from APT. The dose rate used for both computational and experimental data approximately the same $1 \times 10^{-3} \mathrm{dpa} / \mathrm{s}$, though the temperature used in our simulation was considerably higher (1800 K compared to $773 \mathrm{~K}$ in the experiment). The temperature in our simulation was chosen to accelerate the defect diffusion and reduce computational expenditure. The total dose received by the material in our simulation was just $0.1 \mathrm{dpa}$ after $100 \mathrm{~s}$, but the defects introduced are much more mobile. The increased mobility could explain why at longer times the Re concentration is higher than in the experimental results.

There is competition between the transport of Re to the sink and the thermodynamic driving force for dissolution. Without a flux of defects to the sink, our model predicts the dissolution of the Re cluster back into solution, in agreement with the phase diagram. This would imply that if an irradiated W-Re sample were to be annealed, the clusters would dissolve back into solution. This means that in order to facilitate cluster growth there needs to be a continuous flux of Re towards a sink. Both the mixed interstitials and the vacancies bind to $\mathrm{Re}$ and therefore drag it towards the sink. The mixed interstitial is also comprised of two atoms, one of which is a Re, so just by moving to the sink it transports Re to it, and very rapidly due to its $3 \mathrm{D}$ motion and low activation energy for migration. In order to facilitate the nucleation of clusters without the introduction of a sink it might therefore be necessary to use a much higher dose so that the probability of two mixed interstitials binding is increased.

Figure 9 shows the growth of the cluster shown in Figure 7 as a function of time. A cluster is defined as the region within which the Re concentration is above $5 \%$

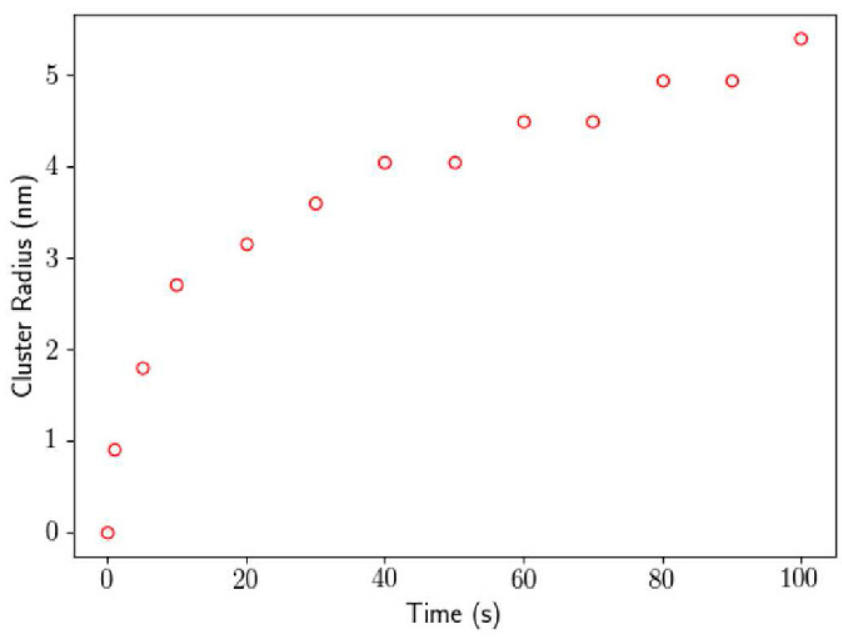

Fig. 9. Growth of cluster in terms of the number of atoms in the cluster as a function of irradiation time for the simulation shown in Figure 7.

which we approximate as spherical. Plotted in Figure 9 is the radius beyond which the concentration drops below $5 \%$ as a function of the irradiation time in seconds. We see the continuous growth of the cluster from the moment the sink is introduced at time $t=0$. As time progresses up to $100 \mathrm{~s}$, there is a slight decrease in the growth rate as more Re from the bulk is contained within the cluster. Overall the growth of the cluster is slower than observed by Huang et al. but begins to approach a similar size after 100 s. In Huang et al. simulations the cluster reaches its maximum size of around $4 \mathrm{~nm}$ after only $20-30 \mathrm{~s}$. In our simulations the cluster reaches $4 \mathrm{~nm}$ after around $40-50 \mathrm{~s}$ but does not stop growing at that point. The growth after this point is reached is slowed but still progresses. This could be due to the fact that the Re concentration in the cluster does not increase as rapidly in our simulations, as shown in Figure 8 where the central concentration reaches only $30 \%$ after $100 \mathrm{~s}$ compared to $50 \%$ in Huang et al. simulations. Compared to Xu et al.'s experiments we see a similarly sized Re clusters to the ion implantations conducted at $773 \mathrm{~K}[6]$.

\subsubsection{Grain boundary segregation}

The potential for segregation of Re to grain boundaries is an important consideration as this has the potential to cause localised embrittlement and premature failure through the degradation of fracture toughness. This segregation has been observed experimentally in polycrystalline $\mathrm{W}$ with a post irradiation composition of $\mathrm{W}-1.4 \mathrm{Re}-0.1 \mathrm{Os}$ [9]. Segregation is expected, but has yet to be observed in ion implanted tungsten, however, we present APT data that confirms this result later in Section 4.3.

Computationally, we study this process by introducing a planar ideal sink into our simulation cell. As before, this feature acts as a perfect sink, removing defects instantaneously if they come with the recombination range of the sink (3NN). In this simplified model there is no crystallographic variation on either side of the GB. In this study we simulate the segregation in a W-1.4Re alloy, in a $2 \times 64 \times 64 \times 256$ atom simulation cell, with a GB placed 


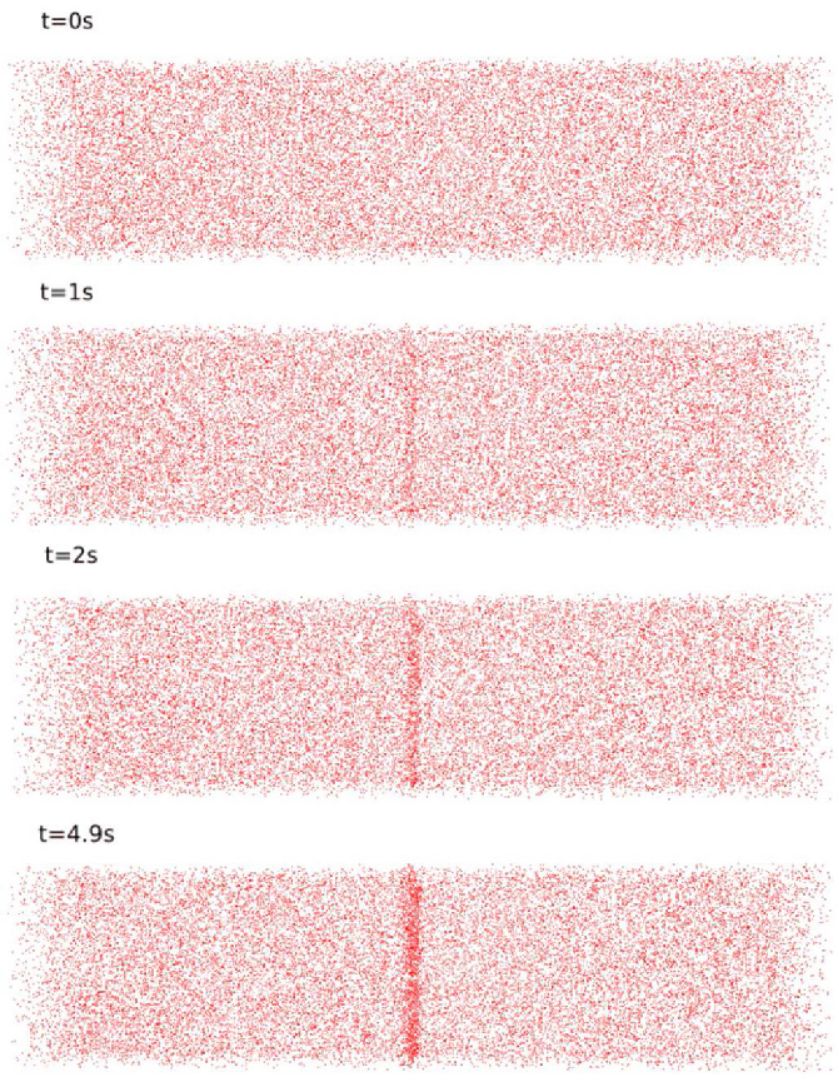

Fig. 10. Segregation of Re in a 1.4 at.\%Re allot to a planar ideal sink at $1800 \mathrm{~K}$ after a total dose of $5.96 \times 10^{-3}$ dpa with a dose rate of $1.19 \times 10^{-3} \mathrm{dpa} / \mathrm{s}$.

perpendicular to the long side of the cell. Defects are introduced at a rate of $1.19 \times 10^{-3} \mathrm{dpa} / \mathrm{s}$ and a temperature of $1800 \mathrm{~K}$ is used to accelerate diffusion. Computational expenditure limited the total simulated time to $5 \mathrm{~s}$, in which a total dose of $5.96 \times 10^{-3} \mathrm{dpa} / \mathrm{s}$ was received.

As expected, the segregation of Re to the point sink was observed. Figure 10 shows the Re atoms in the simulation cell in red with the other atoms hidden. There is clear segregation of Re to the central GB even after only 5 seconds of exposure. The maximum Re concentration at the GB was approximately 8 at.\%Re after 5 seconds of irradiation. Most ion irradiation experiments are limited to temperatures below around $1200 \mathrm{~K}$. As with the point sink simulations, lower temperatures significantly increases computational expenditure as individual defects become trapped or slowed by Re atoms. At higher temperatures the detrapping of these defects is enhanced and the defects reach the sink more quickly. At lower temperatures however, this detrapping is unlikely, and a significant amount of time is spent simulating the defects diffusing within the potential well. These results are discussed in comparison to experimental data in Section 4.3.

\section{Experimental results}

Ion irradiation is a valuable experimental tool as it allows compositions to be studied systematically with a greater degree of control over the composition than is available in neutron irradiation studies. Charged ions deposit their energy over a much shorter distance, resulting in a shallow implanted layer at the surface of the material. The experimental work analysed in this study represents part of a systematic study of the effects of Re and Os on the properties and irradiation induced segregation behaviour of $\mathrm{W}$. We focus on 2 ion implanted samples using APT, a W1.4at.\%Re and a W-25at.\%Re sample. The $\mathrm{W}-1.4 a t . \%$ Re sample was fabricated in order to match the post irradiation composition of the neutron irradiated $\mathrm{W}$ sample from the Extremat II program, studied using STEM-HAADF and APT $[9,13]$. The primary focus of the work presented was to compare the size and composition of Re precipitation under ion irradiation to KMC results for bulk clustering and at GBs.

\subsection{Ion implantation}

Samples from 2 previous ion implantation studies were analysed in this research. W-1.4Re and W-25Re samples were produced by vacuum arc melting which resulted in a very high cooling rate and a coarse grain structure. They were prepared for ion implantation by grinding and polishing with a series of successively finer grit $\mathrm{SiC}$ grinding papers, diamond suspension and colloidal silica. Ion implantation was used to introduce point defects into the samples and produce an implanted surface region. The damage as a function of depth into the sample was estimated using the Stopping Range of Ions in Matter (SRIM) [27] model and a displacement threshold energy of $68 \mathrm{eV}$.

1. W-1.4\%Re was irradiated at the Joint Accelerators for Nano-Science and Nuclear Simulation (JANNuS) facility at CEA Saclay by R. G. Abernethy. The samples were implanted with $\mathrm{W}^{9+}$ ions accelerated to $24 \mathrm{MeV}$ at a temperature of $1073 \mathrm{~K}$. Aluminium degrader foils of $0.8,2$ and $3 \mu \mathrm{m}$ were used to flatten the irradiation profile to produce a more uniform distribution of dpa through the material, as shown in the SRIM output in Figure 11. The average ion flux was $7.0 \times 10^{14} \mathrm{~m}^{-2} \mathrm{~s}^{-1}$ for $11580 \mathrm{~s}$. A dose rate of $1.4 \times 10^{-4} \mathrm{dpa} / \mathrm{s}$ was used with beam intensity, and temperature control measurements carried out according to the procedure described in [28].

2. W-25\%Re was implanted at the Tandem High Energy Implanter at the National Ion Beam Centre, University of Surrey by Alan Xu et al., full details of which are available in $[6,7]$. Samples were mounted to a stainless steel rail using screws and then attached to the heater. The sample was irradiated at $773 \mathrm{~K}$ using a flux of $2.9 \times 10^{14} \mathrm{~cm}^{-2} \mathrm{~s}^{-1}$ $\left(3.82 \times 10^{-4} \mathrm{dpa} \mathrm{s}^{-1}\right) 2 \mathrm{MeV} \mathrm{W} \mathrm{W}^{+}$, ions. Peak damage was estimated as $33 \mathrm{dpa}$ at a depth of approximately $125 \mathrm{~nm}$, with a maximum implantation depth of $300 \mathrm{~nm}$. Temperature was measured using a thermocouple attached to the sample holder and dose rate was measured using Faraday cups. 


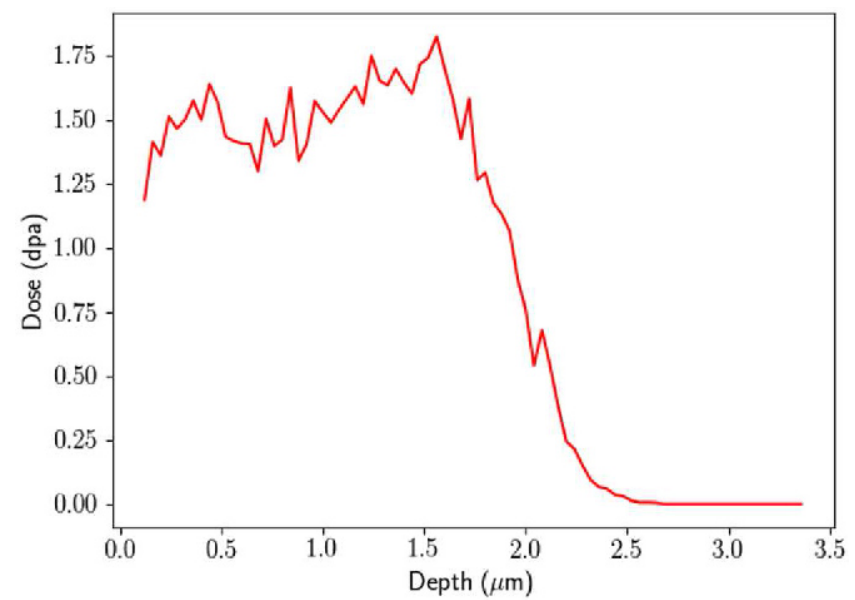

Fig. 11. Dose as a function of depth into the sample for the W-1.4Re sample, estimated using SRIM using a displacement threshold energy of $68 \mathrm{eV}$.

\subsection{Atom probe tomography}

Samples were prepared for Atom Probe Tomography (APT) using the Focused Ion Beam (FIB) liftout technique using a Zeiss NVision 40 dual beam FIB-SEM. A protective region $(15 \mu \mathrm{m} \times 1.5 \mu \mathrm{m})$ of $\mathrm{C}$ was deposited on the surface of the samples using a Gas Injection System (GIS), and for site specific liftouts was aligned along a grain boundary. The region was undercut using a beam current of $1.5 \mathrm{nA}$ and an accelerating voltage of $30 \mathrm{kV}$. The cantilever was lifted out and mounted onto a 22 post silicon coupon, and sharpened into a tip roughly $100 \mathrm{~nm}$ in diameter using an annular milling pattern and a series of successively smaller radii and beam currents between $1.5 \mathrm{nA}$ and $40 \mathrm{pA}$ at $30 \mathrm{kV}$. Once sharpened, the tips received a final polishing stage at $2 \mathrm{kV}, 300 \mathrm{pA}$ to remove Ga implanted material from the FIB beam, removing approximately $50-100 \mathrm{~nm}$ of material. In the case of the W-25\%Re this ensured that the data was taken at the peak of the implantation.

APT was carried out in laser mode in a CAMECA ${ }^{\circledR}$ LEAP $5000 \mathrm{XR}$ using a sample temperature of between 50 and $55 \mathrm{~K}$, and laser pulse energy of $125-150 \mathrm{pJ}$. For the $\mathrm{W}-1.4$ Re sample a pulse frequency of $100 \mathrm{kHz}$ whereas for the W-25Re sample a pulse frequency of $200 \mathrm{kHz}$ was used. The data was reconstructed and visualised using IVAS ${ }^{\circledR}$ 3.8.4. The laser energy was ran at a high energy in order to maximise sample yield without degrading the quality of the mass spectrum due to peak broadening.

\subsection{Re segregation}

In order to analyse a GB in the $\mathrm{W}-1.4 \mathrm{Re}$ sample, targeted sample preparation was used to locate the GB within the tip of the needle. Run parameters were chosen to optimise the ion yield as the samples were highly prone to fracture. Out of 6 prepared samples, one successfully ran for long enough to generate usable data and contained a GB. Figure 12 shows the reconstructed sample with the $\mathrm{W}$ atoms shown in blue and the Re atoms shown in red. The

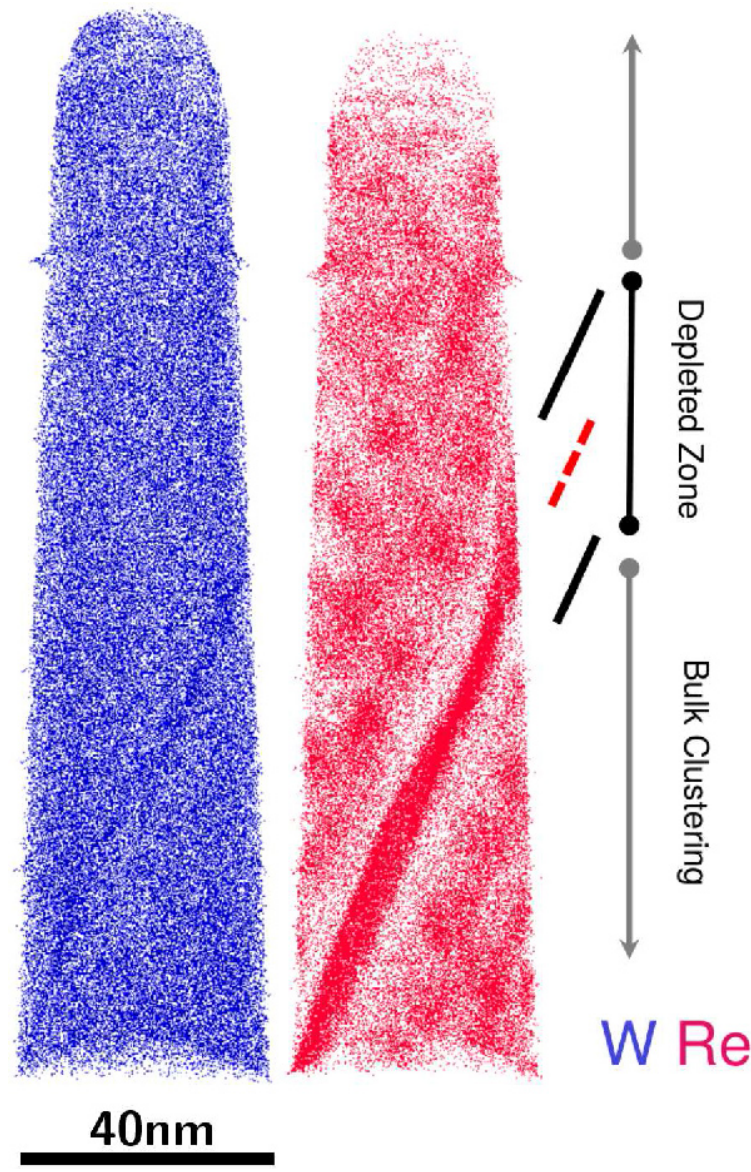

Fig. 12. APT reconstruction of $\mathrm{W}-1.4$ Re sample ion irradiated to $1.67 \mathrm{dpa}$ at $1073 \mathrm{~K}$. Re atoms shown in Red, all other atoms hidden. Grain boundary visible decorated with Re surrounded by a region depleted in Re compared to the bulk. An increase in the W signal also highlights the GB and is a result of artefacts from the APT process.

GB is clearly highlighted by the Re which has segregated to it, but can also be seen in the $\mathrm{W}$ data. There is an apparent increase in the $\mathrm{W}$ density along the GB, as a result of artefacts introduced by the APT process. As the evaporation of atoms from the sample reaches the GB, there is an increase in the number of emitted ions because of the lower field emission required at the GB.

To compare this result with the KMC simulations discussed in Section 3.2.3, the concentration of Re at the GB was measured. A cuboid shaped region of interest was placed over the GB and the concentration was measured in IVAS using a 1D fixed bin width profile. The concentration profile in both the APT and KMC simulation is shown in Figure 13. There is a peak Re concentration of between 4 and 5 at.\% in the APT data, compared to around 8 at.\% in the KMC simulation. There is a depleted zone around the GB, around $10-20 \mathrm{~nm}$ in width, in the APT results that can be seen in both Figures 12 and 13. Away from the GB there is agglomeration of the Re atoms into diffuse clusters. The cluster interface is less pronounced than those observed in previous studies by Xu et al. [6,7] where roughly spherical precipitates were observed. 


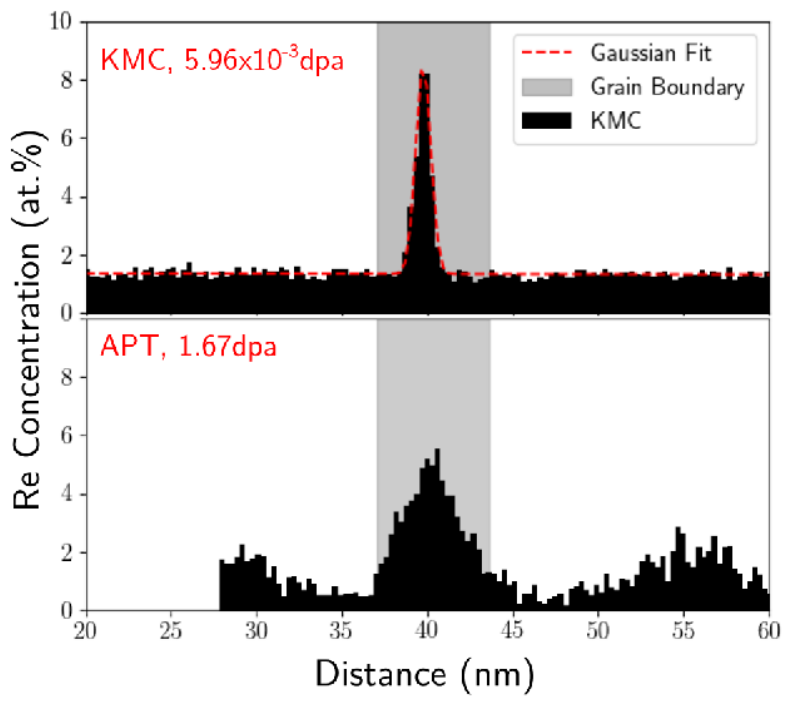

Fig. 13. Segregation of Re in a 1.4 at.\%Re allot to a planar ideal sink at $1800 \mathrm{~K}$ after a total dose of 5.83 dpa with a dose rate of $1.19 \mathrm{dpa} / \mathrm{s}$.

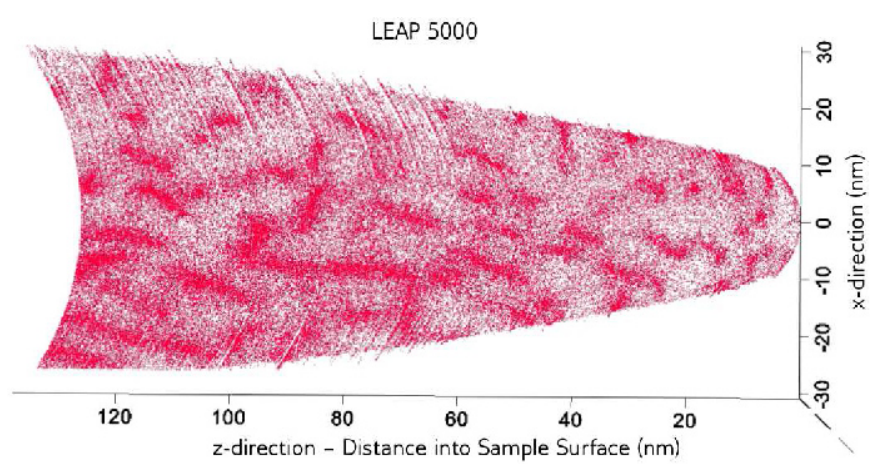

Fig. 14. APT reconstruction of W-25Re sample ion irradiated to $33 \mathrm{dpa}$ at $773 \mathrm{~K}$. Re atoms shown in red, all other atoms hidden.

As shown in Figure 13, in both the simulation and the APT experiments, there is a strong segregation of Re to the GB. The enriched region is wider and has a lower peak concentration in the APT results compared to the simulation. The simulation was only ran for 5 seconds and therefore has a much lower overall dose than the experiments. The higher temperature increases the mobility of the defects, which are responsible for the movement of Re because of their attractive binding energy. Therefore a higher enrichment of the GB is seen in the simulation despite the lower overall dose.

APT of the W-25Re sample revealed the formation of rod shaped precipitates rather than the typically spherical precipitates usually observed in ion implanted W-Re. Figure 14 shows the reconstruction of the W-25Re sample, with only the Re atoms shown. The formation of lenticular precipitates is not usually observed in ion implanted W-Re, but has been seen experimentally in several neutron irradiation studies. Neutron irradiation experiments typically result in a high Re content as $\mathrm{W}$ has a higher absorption cross section for lower energy neutrons, compared to the neutrons produced by a fusion reactor. Several studies have shown the formation of rod/needle shaped Re rich precipitates which are typically associated with chi or sigma phase formation $[12,29]$. Under ion irradiation, samples with lower Re content typically tend to form clusters roughly spherical in shape that are though to be a precursor to precipitation [6,7]. The size and shape of the clusters appears to vary with implantation depth, with smaller clusters being observed nearer to the surface of the sample.

\section{Conclusions}

In this research, a Monte Carlo model, implemented into the open source code, SPPARKS, based on pairwise interactions constructed using the broken-bond model and parameterised using values from DFT calculations is presented. Kinetic Monte Carlo simulations show the segregation of Re to sinks and the formation of clusters that have previously been observed experimentally. We see a central cluster concentration of 29 at.\%Re in a $\mathrm{W}-2$ at.\%Re alloy after $100 \mathrm{~s}$ at of irradiation with a dose rate of $1 \times 10^{-3} \mathrm{dpa} / \mathrm{s}$ at temperature of $1800 \mathrm{~K}$, resulting in a total dose of $0.1 \mathrm{dpa}$. Though the conditions are different, this is similar to the concentration observed experimentally for similar alloy compositions. We also see the decoration of a simulated grain boundary with Re, that we model as a planar ideal sink. We present a preliminary comparison to APT data of a GB in ion implanted W-1.4 at.\%Re, irradiated to $1.67 \mathrm{dpa}$ at $1073 \mathrm{~K}$. Though we see the decoration of the GB both experimentally and computationally, there are key differences which are most likely a result of the different irradiation conditions used. The data obtained in this study is available from [30].

M.J.L.'s work was supported by the UK Engineering and Physical Sciences Research Council [EP/N509711/1] and the Culham Centre for Fusion Energy (CCFE), United Kingdom Atomic Energy Authority through an Industrial CASE scholarship, [Project Reference Number 1802461]. Atom Probe Tomography was carried out at the Oxford Materials Atom Probe Group. D.N-M would like to acknowledge that this work has been carried out within the framework of the EUROfusion Consortium and has received funding from Euratom Research and Training Programme 2019-2020 under Grant Agreement No. 633053 and from the RCUK Energy Programme [grant number EP/P012450/1]. The views and opinions expressed herein do not necessarily reflect those of the European Commission. M.J.L would also like to thank Alan $\mathrm{Xu}$ for preparing the $\mathrm{W}-25 \mathrm{Re}$ sample that was used in this study. E.M. acknowledges support by the U.S. Department of Energy, Office of Science, Office of Fusion Energy Sciences, and Office of Advanced Scientific Computing Research through the Scientific Discovery through Advanced Computing (SciDAC) project on Plasma-Surface Interactions under award number DE-SC0008875. Modelling work was carried out by Matthew J. Lloyd, Duc Nguyen-Manh and Enrique Martinez. Ion implantation experiments were carried out by Robert G. Abernethy. APT experiments were carried out by Matthew J. Lloyd and 
analysed by Matthew J. Lloyd, David E.J. Armstrong, Paul A.J. Bagot and Michael P. Moody.

\section{Authors contribution statement}

All the authors were involved in the preparation of the manuscript. All the authors have read and approved the final manuscript.

Open Access This is an open access article distributed under the terms of the Creative Commons Attribution License (http://creativecommons.org/licenses/by/4.0), which permits unrestricted use, distribution, and reproduction in any medium, provided the original work is properly cited.

\section{References}

1. M. Rieth, J. Nucl. Mater. 432, 482 (2013)

2. D. Armstrong, P. Edmondson, S. Roberts, Appl. Phys. Lett. 102, 251901 (2013)

3. R. Abernethy, Mater. Sci. Technol. 33, 388 (2017)

4. M. Gilbert, J.-C. Sublet, Nucl. Fusion 51, 043005 (2011)

5. M. Gilbert, J.-C. Sublet, S. Dudarev, Nucl. Fusion 57, $044002(2017)$

6. A. Xu, C. Beck, D.E. Armstrong, K. Rajan, G.D. Smith, P.A. Bagot, S.G. Roberts, Acta Mater. 87, 121 (2015)

7. A. Xu, D.E. Armstrong, C. Beck, M.P. Moody, G.D. Smith, P.A. Bagot, S.G. Roberts, Acta Mater. 124, 71 (2017)

8. S. Maloy, M. James, W. Sommer Jr., G. Willcutt Jr., M. Lopez, T. Romero, M.B. Toloczko, J. Nucl. Mater. 343, 219 (2005)

9. M. Klimenkov, U. Jäntsch, M. Rieth, H. Schneider, D. Armstrong, J. Gibson, S. Roberts, Nucl. Mater. Energy 9, $480(2016)$

10. A. Hasegawa, M. Fukuda, S. Nogami, K. Yabuuchi, Fusion Eng. Des. 89, 1568 (2014)

11. Y. Katoh, L.L. Snead, L.M. Garrison, X. Hu, T. Koyanagi, C.M. Parish, P.D. Edmondson, M. Fukuda, T. Hwang, T. Tanaka, A. Hasegawa, J. Nucl. Mater. 520, 193 (2019)
12. X. Hu, C.M. Parish, K. Wang, T. Koyanagi, B.P. Eftink, Y. Katoh, Acta Mater. 165, 51 (2019)

13. M.J. Lloyd, R.G. Abernethy, M.R. Gilbert, I. Griffiths, P.A.J. Bagot, D. Nguyen-Manh, M.P. Moody, D.E.J. Armstrong, Scr. Mater. 173, 96 (2019)

14. F. Soisson, C.-C. Fu, Phys. Rev. B 76, 214102 (2007)

15. F. Soisson, C. Becquart, N. Castin, C. Domain, L. Malerba, E. Vincent, J. Nucl. Mater. 406, 55 (2010)

16. E. Martínez, O. Senninger, C.-C. Fu, F. Soisson, Phys. Rev. B 86, 224109 (2012)

17. O. Senninger, F. Soisson, E. Martínez, M. Nastar, C.-C. Fu, Y. Bréchet, Acta Mater. 103, 1 (2016)

18. E. Martínez, O. Senninger, A. Caro, F. Soisson, M. Nastar, B.P. Uberuaga, Phys. Rev. Lett. 120, 106101 (2018)

19. C.-H. Huang, L. Gharaee, Y. Zhao, P. Erhart, J. Marian, Phys. Rev. B 96, 094108 (2017)

20. T. Suzudo, M. Yamaguchi, A. Hasegawa, Modell. Simul. Mater. Sci. Eng. 22, 075006 (2014)

21. T. Suzudo, M. Yamaguchi, A. Hasegawa, J. Nucl. Mater. 467, 418 (2015)

22. T. Suzudo, A. Hasegawa, Sci. Rep. 6, 36738 (2016)

23. J. Wróbel, D. Nguyen-Manh, K. Kurzydłowski, S. Dudarev, J. Phys.: Condens. Matter 29, 145403 (2017)

24. S. Plimpton, C. Battaile, M. Chandross, L. Holm, A. Thompson, V. Tikare, G. Wagner, E. Webb, X. Zhou, C.G. Cardona et al., Sandia Report SAND2009-6226, 2009

25. A. Stukowski, Modell. Simul. Mater. Sci. Eng. 18, 015012 (2009)

26. J. Mundy, S. Rothman, N. Lam, H. Hoff, L. Nowicki, Phys. Rev. B 18, 6566 (1978)

27. J.F. Ziegler, M.D. Ziegler, J.P. Biersack, Nucl. Instrum. Methods Phys. Res. Sect. B 268, 1818 (2010)

28. S. Pellegrino, P. Trocellier, S. Miro, Y. Serruys, É. Bordas, H. Martin, N. Chaâbane, S. Vaubaillon, J. Gallien, L. Beck, Nucl. Instr. Methods Phys. Res. Sect. B 273, 213 (2012)

29. A. Hasegawa, M. Fukuda, K. Yabuuchi, S. Nogami, J. Nucl. Mater. 471, 175 (2016)

30. M.J. Lloyd, M.P. Moody, D.E.J. Armstrong, D. Nguyen-Manh, Oxford Research Archive (2019), DOI: 10.5287/bodleian:G2YDAv10p 